\title{
Scene complexity: influence on perception, memory, and development in the medial temporal lobe
}

\author{
Xiaoqian J. Chai ${ }^{1+}$, Noa Ofen ${ }^{1 *}{ }^{*}$, Lucia F. Jacobs ${ }^{2}$ and John D. E. Gabrieli ${ }^{1}$ \\ Department of Brain and Cognitive Sciences, Massachusetts Institute of Technology, Cambridge, MA, USA \\ 2 Department of Psychology, University of California Berkeley, Berkeley, CA, USA
}

Edited by:

Silvia A. Bunge, University of California Berkeley, USA

Reviewed by:

Simona Ghetti, University of California, USA

Amanda Guyer, University of California, USA

\section{*Correspondence:}

Noa Ofen, Brain and Cognitive

Sciences Department, Massachusetts

Institute of Technology, Cambridge, MA

02139, USA.

email:noa@mit.edu

${ }^{+}$Xiaoqian J. Chai and Noa Ofen contributed equally to the paper.
Regions in the medial temporal lobe (MTL) and prefrontal cortex (PFC) are involved in memory formation for scenes in both children and adults. The development in children and adolescents of successful memory encoding for scenes has been associated with increased activation in PFC, but not MTL, regions. However, evidence suggests that a functional subregion of the MTL that supports scene perception, located in the parahippocampal gyrus (PHG), goes through a prolonged maturation process. Here we tested the hypothesis that maturation of scene perception supports the development of memory for complex scenes. Scenes were characterized by their levels of complexity defined by the number of unique object categories depicted in the scene. Recognition memory improved with age, in participants ages 8-24, for high-, but not low-, complexity scenes. High-complexity compared to low-complexity scenes activated a network of regions including the posterior PHG. The difference in activations for high-versus low-complexity scenes increased with age in the right posterior PHG. Finally, activations in right posterior PHG were associated with age-related increases in successful memory formation for high-, but not low-, complexity scenes. These results suggest that functional maturation of the right posterior PHG plays a critical role in the development of enduring long-term recollection for high-complexity scenes.

Keywords: declarative memory, perception, cognitive development, visual scenes, fMRI, parahippocampal gyrus

\section{INTRODUCTION}

The neural systems mediating declarative or explicit memory for facts and events in adults have been identified through convergent lesion and functional neuroimaging evidence. Medial temporal lobe (MTL) structures, including the hippocampus and surrounding perirhinal and parahippocampal cortices, are essential for the formation of new declarative memories. Bilateral MTL injury results in global amnesia, defined by an inability to form new declarative memories (Scoville and Milner, 1957; Zola-Morgan et al., 1986; Squire, 1992). Prefrontal cortex (PFC) is not essential for memory formation, but PFC lesions impair declarative memory for contextual details of an experience (source memory) (Schacter et al., 1984; Janowsky et al., 1989). Functional neuroimaging studies in healthy adults have found that greater magnitudes of PFC and MTL activation during encoding correlate, on a stimulus-by-stimulus or event-related basis, with successful memory formation as evidenced by accurate subsequent memory for scenes, words, and faces (Brewer et al., 1998; Wagner et al., 1998; Buckner et al., 1999; Davachi et al., 2003; Nichols et al., 2006).

Compared to the large knowledge about the neural correlates of memory in adults, little is known about the normal development of declarative memory systems from childhood through adulthood (Menon et al., 2005; Chiu et al., 2006; Ofen et al., 2007; Paz-Alonso et al., 2008). Behavioral evidence suggests that declarative memory develops from childhood through adulthood, and that development is most protracted for memory tasks that demand greater detail in recollection, such as remembering specific details of the context in which information was presented, relative to the information itself
(Dirks and Neisser, 1977; Mandler and Robinson, 1978; Cycowicz et al., 2001; Billingsley et al., 2002; Ghetti and Angelini, 2008). The developmental distinction between different aspects of recognition memory may reflect two dissociable mnemonic processes; recollection, that includes specific knowledge of the details of the event, and familiarity, that may include less contextual details (Jacoby et al., 1993; Yonelinas, 1999). In a functional neuroimaging study of memory encoding for scenes in 8 to 24-year olds, development of successful memory encoding was associated with increased activation in PFC regions that were specifically associated with recollection (but not familiarity) for scenes (Ofen et al., 2007). In contrast, MTL regions were similarly activated across age. These findings suggested that the protracted development of recollection relative to familiarity could reflect protracted maturation of PFC relative to MTL functions supporting memory formation.

The lack of developmental changes for activations in the MTL in our previous study suggested that memory processes served by MTL are mature by the age of 8 years. This conclusion, however, contradicts other evidence that implicates prolonged maturation of MTL subregions. First, memory for high-level visual stimuli such as natural scenes and faces grows from childhood through adolescence into young adulthood (Diamond and Carey, 1977; Mandler and Robinson, 1978), and this development of memory ability has been associated with the development of cortical areas that are specialized for visual perception of scenes and faces (Golarai et al., 2007). Specifically, functionally-defined scene-selective parahippocampal cortex known as the 'parahippocampal place area' or PPA (Epstein and Kanwisher, 1998) grows in size from childhood through adulthood, and this 
growth in size correlates with recognition memory for scenes. These findings suggest that prolonged maturation of at least parts of the MTL ought to be associated with prolonged maturation of some aspects of memory for scenes. Second, post-mortem and structural imaging evidence indicates that PFC maturation appears to continue into late adolescence (Huttenlocher, 1979; Giedd et al., 1999; Gogtay et al., 2004; Sowell et al., 2004), but it also suggests continued development of MTL regions in the human brain (Giedd et al., 1996; Sowell and Jernigan, 1998; Sowell et al., 2002; Gogtay et al., 2006). These anatomical findings support the idea that PFC memory functions develop more slowly than MTL memory functions in the human brain, but also raise the possibility that functional developmental may occur in the MTL in the 8 to 24 -year-old range.

Here, we examined the effect of visual scene complexity on three related issues. First, we tested whether scene complexity modulates the magnitude of brain activation in scene-perception regions. Second, we tested whether scene complexity effects undergo prolonged maturation. Third, we tested whether scene complexity influences the development of memory, and MTL functions associated with memory formation. Complexity of real-world scenes is represented by multiple dimensions including the quantity of objects in the scene (Oliva et al., 2004). When regularities are present in a scene, e.g., identical objects, the visual pattern is simplified by perceptual grouping (Feldman, 1997). Thus, a scene picturing a forest may depict many trees, but perceptual grouping allows the trees to be perceived as a single group. A scene picturing a typical living room or kitchen, however, may depict a variety of distinct objects. To account for the effect of grouping, we characterized scene complexity as the number of unique object categories in the scene. We hypothesized that processing more complex scenes will likely result in greater activations in scene-selective regions in the parahippocampal gyrus (PHG). We further hypothesized that processing of more complex scenes will have protracted maturation. These effects in turn may be associated with improvement in memory ability with age. Thus the third hypothesis tested here is that developmental effects of MTL functions associated with memory formation will be more pronounced for more complex scenes. Accurate recognition memory for more complex scenes may be limited in children who have an immature functionally-defined scene-selective PHG (Golarai et al., 2007). Children may not have the mature representational capacities needed for the encoding of detailed specificity found in more complex scenes. This less than optimal representation at encoding will then limit successful retrieval at test. Such an effect of complexity may have been obscured in the prior study (Ofen et al., 2007) because half the scenes were outdoor pictures with low visual complexity (trees, lakes, mountains, etc.).

We also examined whether a low-level visual property of the scenes, spatial frequency, influenced our findings because highcomplexity scenes have higher spatial frequencies than lowcomplexity scenes. Neurophysiological (De Valois et al., 1982; Foster et al., 1985) and fMRI studies (Vuilleumier et al., 2003; Iidaka et al., 2004; Peyrin et al., 2004) suggest that information associated with different spatial frequencies is represented in different brain regions. We therefore measured the mean spatial frequency in each scene and examined whether spatial frequency influenced the effects of scene complexity on activations in regions involved in scene perception, the maturation effects in these regions and the influence of scene complexity on measures of memory formation.
We present the results of a reanalysis of previously published data (Ofen et al., 2007) that examined the normal development of activations related to successful memory formation, as measured by functional magnetic resonance imaging (fMRI), in healthy children, adolescents and adults from age 8 to 24 . Participants viewed indoor and outdoor scenes during a scanned study phase. Afterwards, they received a recognition memory test with the previously studied scenes and new (foil) scenes. For scenes judged as having been seen at study, participants rated their memories as "remembered" (R responses which indicated a vivid memory accompanied by details of the experience) or "familiar" (K responses which indicated that participants know they have studied the scene, but that the memory was not accompanied by details of the experience). We coded all scenes for complexity, and reanalyzed the behavioral and imaging data to examine the effects of complexity. We tested the effects scene complexity on (1) activations in regions involved in scene perception (2) functional maturation in scene-perception regions, and (3) the development of memory formation via behavior and MTL activation.

\section{MATERIALS AND METHODS MATERIALS}

A total of 500 full-color photographs of natural scenes (250 indoor, 250 outdoor) were used, and randomly divided into ten lists of 50 pictures. Lists were counterbalanced across participants, such that all lists were presented equally often as study and test items across participants. The number of unique object categories for each scene was calculated as an index for scene complexity using the LabelMe image toolbox (Russell et al., 2008) (Figures 1A,B). Scenes that included three or fewer object categories were classified as low-complexity scenes, and scenes that included five or more object categories were classified as high-complexity scenes. Out of the 250 studied scenes, $103.7 \pm 1.9$ (mean \pm SD) were low-complexity scenes, and $113.9 \pm 4.4$ were high-complexity scenes. Out of the 250 foils used in the unscanned recognition test, $103.3 \pm 1.9$ were low-complexity scenes, $113.1 \pm 4.4$ were high-complexity scenes. Scenes with four object categories were classified as mid-level complexity scenes $(32.4 \pm 6.0$ studied scenes, and $33.6 \pm 6.0$ foils). Results are presented for the highand low-complexity scenes.

To characterize the spatial frequency for each scene, the power spectrum of the scene was calculated by performing a $2 \mathrm{D}$ discrete Fourier transform on the image. The power spectrum can be modeled as a function of frequency $f$ and orientation $\theta$. The frequency distribution at different orientations in the image varies for different types of scenes (Torralba and Oliva, 2003). Here we considered eight different orientations: horizontal, vertical, and six oblique orientations. The power spectrum for each scene was fitted to a linear model at the frequency interval $f=\left[\begin{array}{lll}0.01 & 0.45\end{array}\right]$ cycles/pixel for each of the eight orientations $\theta=[0 \pi]$. The average slope and intercept of the fitted line for the 8 orientations were used to characterize the frequency distribution of the scene. High-complexity scenes had significantly higher spatial frequencies than low-complexity scenes $[t(432)=3.43, p<0.001]$. We therefore included spatial frequency for each scene as a parametric modulator in the imaging data analysis to account for effects associated with spatial frequency ( $f M R I$ data analysis section). 


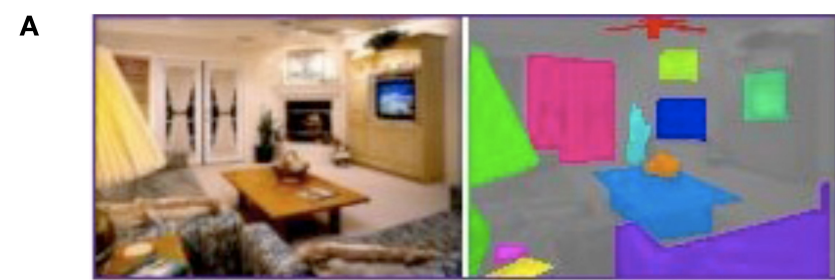

C

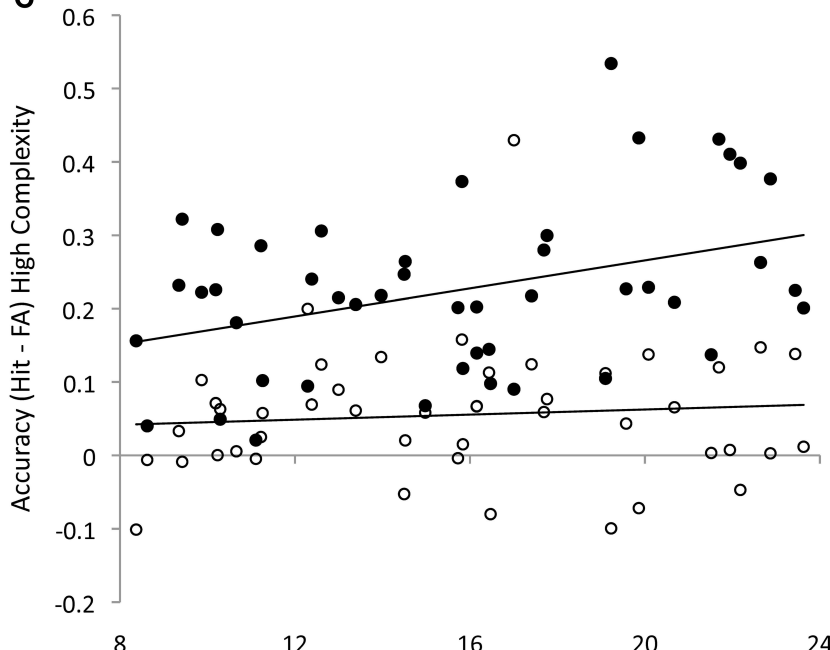

Age (years)
B

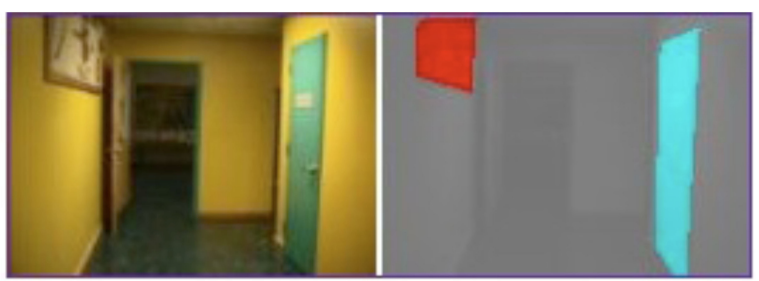

D

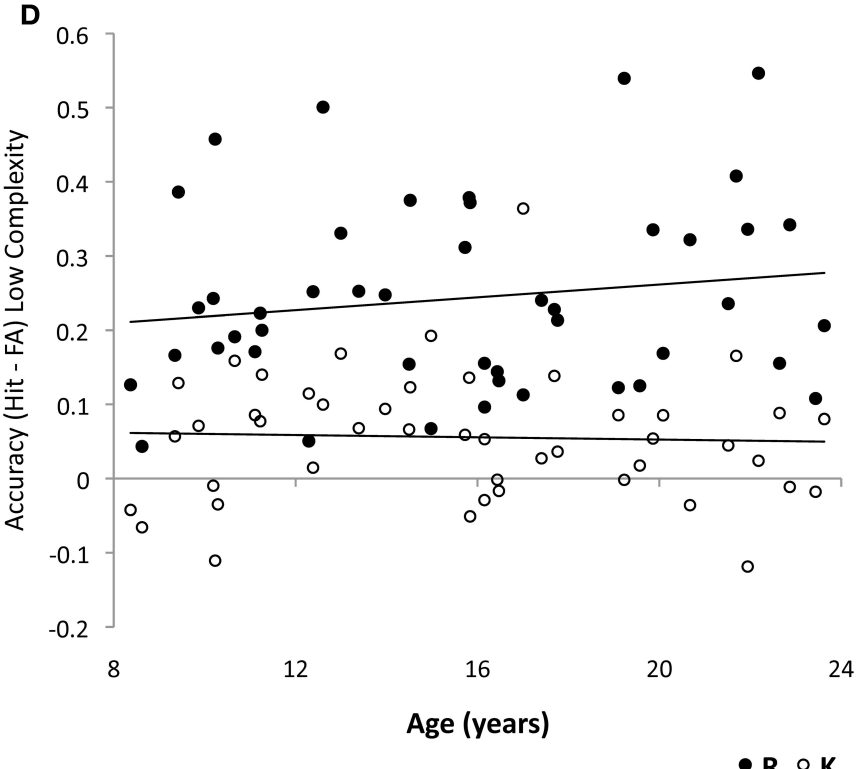

recollection rates $\left(\mathrm{R}_{\mathrm{HIT}}-\mathrm{R}_{\mathrm{FA}}\right)$ (filled circles) and adjusted familiarity rates $\left(\mathrm{K}_{\mathrm{HIT}}-\right.$ $\mathrm{K}_{\mathrm{FA}}$ ) (open circles) for (C) high-complexity scenes and (D) low-complexity scenes plotted against the participants' age. Recollection for low-complexity scenes and familiarity for high- and low-complexity scenes did not change reliably with age.

\section{PARTICIPANTS}

Fifty-two volunteers, ages 8-24 years, were recruited from the Stanford University community and provided informed consent as indicated by a Stanford University IRB-approved protocol. All participants were right-handed, had normal or corrected-to-normal vision, with no history of psychiatric or neurological disorder. Data are presented for 46 participants ( 24 females). Two participants were excluded as a result of sustained image artifacts (male, age 12; female, age 10). In addition, four participants (males, ages $8,11,13$; female, age 15) were excluded due to incomplete behavioral data.

\section{PROCEDURE}

Participants viewed 125 indoor and 125 outdoor scenes during a scanned study phase that was followed by a recognition memory test. During scanning, each picture was presented for $3 \mathrm{~s}$ with $1 \mathrm{~s}$ of inter-trial interval. Participants made "indoor" or "outdoor" judgments to each scene by pressing a button on the button box. Trials with incorrect or no responses were excluded from the analyses (error trials). The study phase was divided into five sessions, each with 50 scenes. After the scanning session, participants were given a self-paced recognition test of the 250 scenes studied during the scanning session and 250 new scenes. If the participant responded "old" to a scene, they were further asked to indicate if they "actually remembered" the scene $(\mathrm{R})$ or if the scene "just looks familiar" $(\mathrm{K})$. Adjusted memory accuracy was calculated by subtracting the false alarm rate ("old" responses to new pictures) from the hit rate ("old" responses to studied pictures). If a "new" response was given to a studied scene, the trial was classified as a "forgotten" trial (F).

\section{MRI DATA ACOUISITION}

MRI data were acquired in a $1.5 \mathrm{~T}$ GE scanner. Functional images were acquired in 24 slices parallel to the line connecting anterior and posterior commissures, with $2 \mathrm{~s}$ repetition time, $60^{\circ}$ flip angle, $64 \times 64$ voxels, and $3.75 \mathrm{~mm}$ in-plane resolution.

\section{fMRI DATA ANALYSIS}

Functional imaging data were analyzed in SPM5 (Department of Imaging Neuroscience, London, UK). Images were slice timing corrected, motion corrected, spatially normalized to the T2 Montreal Neurological Institute (MNI) template, and smoothed with a 6-mm Gaussian kernel. Data were inspected for artifacts and motion using custom software (http://web.mit.edu/swg/software/). First-level 
analysis was performed with a general linear model (GLM) with regressors for six conditions of interest $(\mathrm{R}, \mathrm{K}$, and $\mathrm{F}$ trial types for low- and high-complexity scenes) and two conditions of no interest (trials from mid-level complexity scenes, and error trials, including trials when no response was recorded). Additional regressors accounted for head movement (three translation, three rotation parameters) and outlier scans (images in which average intensity deviated more than $3 \mathrm{SD}$ from the mean intensity in the session or in which movement exceeded $0.5 \mathrm{~mm}$ or $0.01^{\circ}$ from the previous image). For each participant, contrasts of different trial types were created from linear combination of the GLM regressors. These contrasts included (a) High > Low complexity (for each level of complexity, activation for R, F, K conditions were combined) (b) R $>$ F high complexity and (c) R $>$ F low complexity. These contrasts were entered into a second-level random effects analysis to identify activations on a group level. In order to investigate age differences we conducted a whole brain regression analysis with age as a regressor of interest. Region of interest (ROI) in the posterior PHG was defined functionally as a region where activations increased with age in the contrast High > Low complexity. We extracted contrast estimates of subsequent memory performance from the contrasts $\mathrm{R}>\mathrm{F}$ and $\mathrm{K}>\mathrm{F}$ for both high- and low-complexity scenes from this ROI. These extracted measures from all participants were tested for correlation with age. Unless otherwise mentioned, we report activations that survived a threshold of $p<0.001$ at the voxellevel, corrected at the cluster-level of $p<0.05$. In a second GLM model, spatial frequency of each scene was included as a parametric modulator for each condition to control for the effects of spatial frequency. The same conditions and regressors as described above were computed at the first-level analyses.

\section{RESULTS \\ BEHAVIOR}

Across all participants, $50 \% \pm 13$ of studied high-complexity scene were later recognized as "old" (hit rate), and $52 \% \pm 18$ of studied low-complexity scenes were later recognized as "old". For new scenes presented at test (foils), $22 \% \pm 16$ of high-complexity scenes were falsely categorized as "old" (false alarm rate), and 22\% \pm 14 of low-complexity scenes were falsely categorized as "old". Across all participants, adjusted hit rate (hit rate - false alarm rate) for high-complexity scenes $(28 \% \pm 13)$ did not differ from that for lowcomplexity scenes $(30 \% \pm 14)\left(t_{(45)}=1.02, p=0.31\right)$. Critically, the adjusted hit rate increased with age for high-complexity $(r=0.40$, $p=0.006)$, but not for low-complexity $(r=0.11, p=0.46)$ scenes. Thus, overall participants had similar memory accuracy for highand low-complexity scenes, but memory accuracy improved with age only for high-complexity scenes.

Participants categorized as "remembered" $\left(\mathrm{R}_{\mathrm{HIT}}\right), 28 \% \pm 12$ of the studied high-complexity scenes, and $29 \% \pm 15$ of the studied low-complexity scenes. Participants had similar rates of falsely categorizing as "remembered" $\left(\mathrm{R}_{\mathrm{FA}}\right)$ high $(5 \% \pm 6)$ and low $(5 \% \pm 6)$ complexity foils. Across all participants, adjusted $\mathrm{R}$ rates $\left(\mathrm{R}_{\mathrm{HIT}}-\mathrm{R}_{\mathrm{FA}}\right)$ for high-complexity scenes did not differ from that for low-complexity scenes $\left(t_{(45)}=1.41, p=0.17\right)$. Adjusted $\mathrm{R}$ rates increased with age for high-complexity $(r=0.38, p=0.009)$, but not for low-complexity $(r=0.16, p=0.30)$, scenes (Figures 1C,D). The difference between the adjusted $\mathrm{R}$ rates of high- and low-complex- ity scenes increased with age ( $r=0.27, p=0.03$; 1 -tailed), suggesting that the age-related improvements in memory performance were more robust for recollected high-complexity scenes. Thus, overall, participants had similar memory accuracy for recollected high- and low-complexity scenes, but accuracy for recollected highcomplexity scenes improved with age, whereas accuracy for recollected low-complexity scenes did not change with age.

Participants categorized as "familiar" $\left(\mathrm{K}_{\mathrm{HIT}}\right), 22 \% \pm 9$ of studied high-complexity scenes, and $22 \% \pm 10$ of studied low-complexity scenes. Participants falsely categorized as "familiar" $\left(\mathrm{K}_{\mathrm{FA}}\right) 17 \% \pm 11$ of high-complexity, and 17\% \pm 12 of low-complexity foils. Across all participants, adjusted $\mathrm{K}$ rates $\left(\mathrm{K}_{\mathrm{HIT}}-\mathrm{K}_{\mathrm{FA}}\right)$ for high- complexity scenes did not differ from that for low-complexity scenes $\left(t_{(45)}=0.06, p=0.95\right)$. Adjusted $\mathrm{K}$ rates did not change with age for either high-complexity or low-complexity scenes $(|r| s<0.09, p s>0.56)$ (Figures 1C,D).

During the encoding task, participants were overall faster to respond to low-complexity scenes $(1072 \pm 275 \mathrm{~ms})$ than to highcomplexity scenes $(1093 \pm 287 \mathrm{~ms})\left(t_{(45)}=2.31, p=0.03\right)$. Response times decreased with age for low- $(r=-0.37, p=0.01)$ and high$(r=-0.33, p=0.03)$ complexity scenes. The difference between the response times for high- and low-complexity scenes, however, did not change with age $(r=0.14, p=0.35)$. Response times decreased with age for $\mathrm{R}, \mathrm{K}$ and $\mathrm{F}$ trial types for both high- and low-complexity scenes $(-0.36<r<-0.30$, ps $<0.04)$. The difference between response times for $\mathrm{R}$ and $\mathrm{F}$ trial types, however, did not change with age for either high $(r=0.12, p=0.43)$ or low complexity scenes $(r=0.08, p=0.60)$.

\section{IMAGING}

\section{Scene complexity effects}

Across all participants, there were greater activations for high- than low-complexity scenes in occipital and posterior medial temporal lobe regions (Table 1). To examine the effects of low-level visual properties of the scenes, we constructed a second GLM model in which the spatial frequency was included as a parametric modulator to account for the effects of spatial frequency of the scenes. The same clusters remained significant after the effects of spatial frequencies were accounted for.

\section{Development of scene complexity effects}

A whole-brain regression analysis for High > Low complexity with age as the regressor revealed age-related increases in activations in right posterior PHG and frontal cortex (BA 6) (Table 1). Activations in both of these regions remained significant after the effects of spatial frequency were accounted for.

\section{Development of scene complexity effects that are related to subsequent memory - region of interest analysis}

We further examined subsequent memory effects in the PHG region $(26,-54,-4)$ in which activations for High $>$ Low complexity scenes increased with age (Figure 2). This analysis allowed us to relate developmental changes in processing of high versus low complexity scenes to developmental changes in subsequent memory.

Development of subsequent memory effects for high-complexity scenes. Age-related increases in subsequent memory activations were found only for the contrast $\mathrm{R}>\mathrm{F}$ for high-complexity 
Table 1 |Activations for high- versus low-complexity scenes.

\begin{tabular}{|c|c|c|c|c|c|c|c|}
\hline & & BA & $x$ & $y$ & $z$ & $T$ & \# voxels \\
\hline \multicolumn{8}{|c|}{ HIGH > LOW } \\
\hline Right & Posterior cingulate/precuneus & 31 & 22 & -60 & 20 & 9.64 & 3344 \\
\hline Left & Posterior cingulate/precuneus & 31 & -18 & -60 & 24 & 6.98 & \\
\hline Left & Parahippocampal gyrus & $19 / 37$ & -30 & -42 & -4 & 8.96 & 873 \\
\hline Left & Lingual gyrus & 19 & -26 & -82 & 0 & 4.94 & \\
\hline \multirow[t]{2}{*}{ Right } & Middle occipital gyrus & 18 & 22 & -88 & -4 & 6.61 & 950 \\
\hline & & $18 / 19$ & 34 & -90 & 12 & 6.22 & \\
\hline Right & Medial frontal gyrus & $11 / 10$ & 6 & 52 & -10 & 4.72 & 68 \\
\hline Right & Middle/Inferior temporal gyrus & 21 & 52 & -6 & -18 & 4.41 & 106 \\
\hline
\end{tabular}

Coordinates $(x y z)$ are based on MNI brain (Montreal Neurologic Institute). BA, Brodmann area; $t, t$ value at peak voxel.
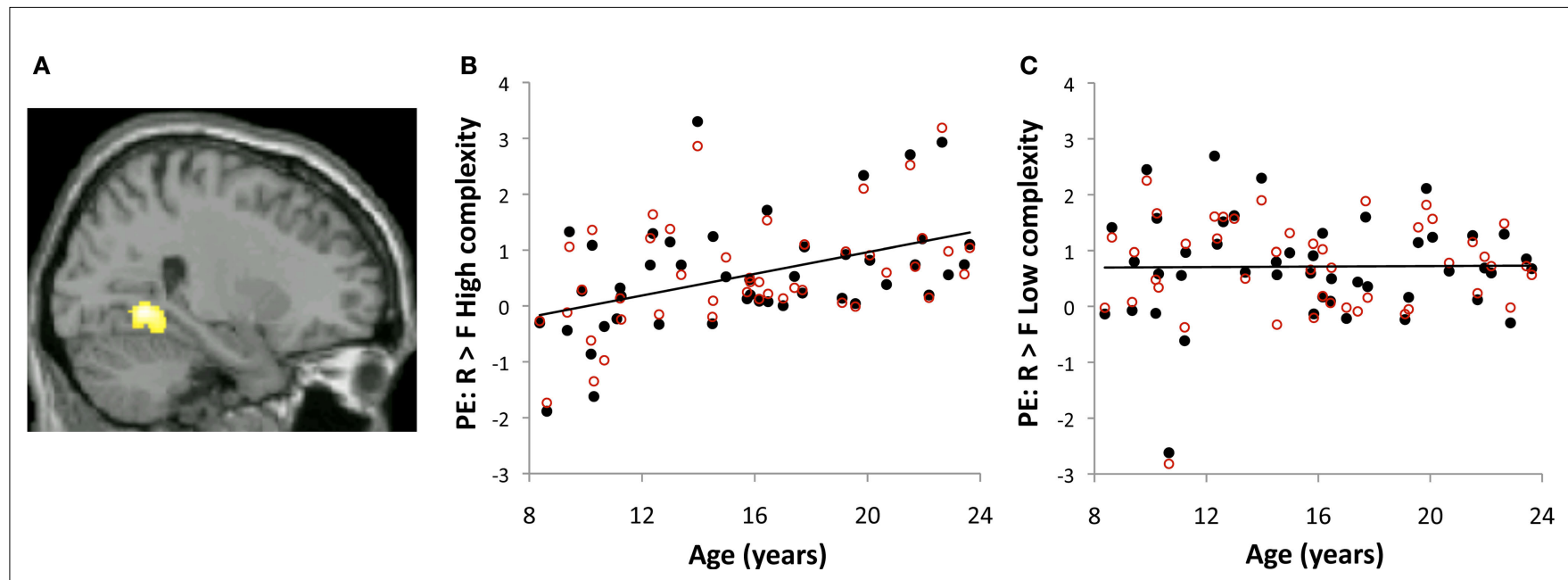

FIGURE 2 |Activations associated with successful memory formation for high- and low- complexity scenes in posterior parahippocampal gyrus.

(A) Axial brain image depicting right posterior parahippocampal $\mathrm{ROI}$ defined by increase in activation with age for high $>$ low complexity scenes. Image corresponds to $\mathrm{MNI} \times$ coordinate 26 . $(\mathrm{B}, \mathrm{C})$ Plots of correlations of the individual parameter estimates (PE) in the contrast $R>F$ for high- (B) and low(C) complexity scenes (black, filled circles). Overlaid in red (open circles) are the individual parameter estimates extracted from contrasts $\mathrm{R}>\mathrm{F}$ for high(B) and low- (C) complexity scenes after correcting for the scene's spatial frequency. scenes $(r=0.44, p=0.002)$. There were no age-related changes in subsequent memory activations for the contrast $\mathrm{R}>\mathrm{F}$ for low complexity scenes $(r=0.01, p=0.94)$. Furthermore, there were no agerelated changes for subsequent memory activations in the contrast $\mathrm{K}>\mathrm{F}$ for either high- $(r=0.02, p=0.89)$ or low- $(r=0.04, p=0.78)$ complexity scenes. The behavioral improvement in memory with age implied that with age, participants remembered more highcomplexity scenes. We thus tested whether age-related increases in subsequent memory activations for high-complexity scenes merely reflect less statistical power to detect subsequent memory activation in younger participants. Age-related increases in subsequent memory activations for the contrast $\mathrm{R}>\mathrm{F}$ for high-complexity scenes remained significant when recognition accuracy (adjusted $\mathrm{R}$ rates) was accounted for $(r=0.35, p=0.02)$, and when the number of remembered high-complexity scenes was accounted for $(r=0.40$, $p=0.007)$. These results suggest that the age-related increase in subsequent memory activations in the right posterior PHG for high-complexity scenes is not the result of reduced statistical power to detect activations in the younger participants, but rather that with age, increased activations for high-complexity scenes, compared to low-complexity scenes, contributed to improvement in memory for high-complexity scenes. 
Development of subsequent memory effects for high-complexity scenes is not affected by low-level visual properties of scenes. We extracted subsequent memory activations in the same PHG ROI described above (Figure 2, red open circles) from results obtained with a second GLM model that took into account the effect of a low-level visual property of the scene, spatial frequency. Similar to results presented above, age-related increases in subsequent memory activations in the PHG ROI were only found for the contrast $\mathrm{R}>\mathrm{F}$ of high-complexity scenes $(r=0.44, p=0.002)$. There were no age-related changes in subsequent memory activations for the contrast $\mathrm{R}>\mathrm{F}$ of low-complexity scenes $(r=0.04, p=0.79)$.
Thus, controlling for spatial frequency did not change the pattern of result in age-related changes in subsequent memory activation, suggesting that these effects were driven by the high-level scene property (image complexity) rather than a low-level visual property (spatial frequency).

\section{Subsequent memory effects as a function of scene complexity}

Subsequent memory for remembered scenes were investigated separately for high- and low-complexity scenes by comparing activations for remembered and forgotten scenes (contrast R > F) (Table 2). Across all participants, activations for subsequent memory of

Table 2 | Subsequent memory activations for remembered scenes.

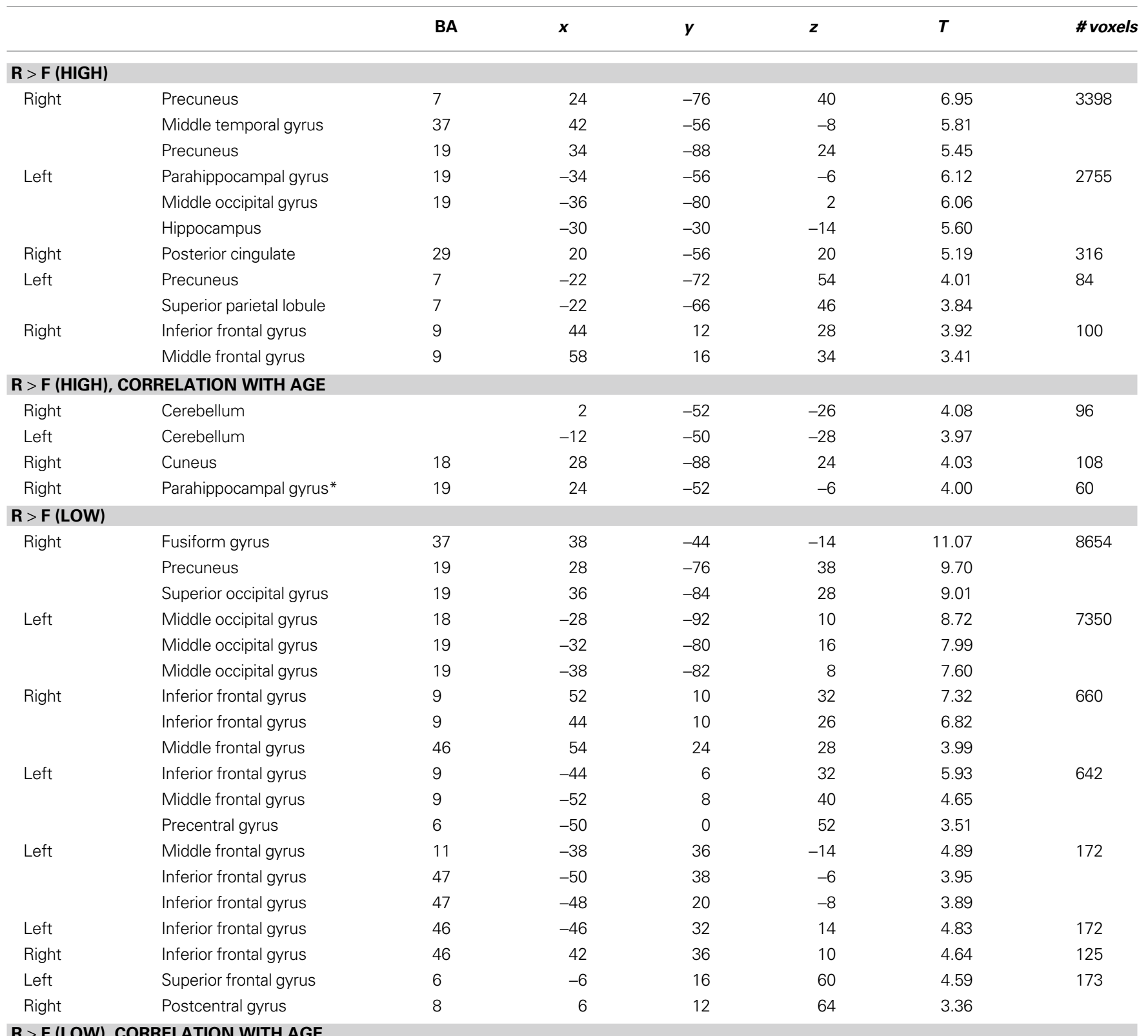

None

${ }^{*} p=0.06$ (cluster-level). 
high-complexity remembered scenes were located in large bilateral posterior clusters spanning the middle occipital cortex, extending ventrally into the middle temporal, fusiform and parahippocampal gyri, and dorsally into the precuneus and superior parietal lobule, and in several PFC regions. There were age-related increases associated with subsequent memory for high-complexity scenes in several regions, including right posterior PHG (Table 2).

Subsequent memory activation for low-complexity remembered scenes across all participants were found in locations similar to those found for remembered high- complexity scenes, (Table 2). These activations, however, did not change with age. Thus, activation in right posterior PHG, an MTL subregion, increased with age for subsequently remembered high-complexity, but not lowcomplexity, scenes (Figure 3).

We also examined subsequent memory for correctly recognized old scenes that were given "familiar" responses (contrast $\mathrm{K}>\mathrm{F}$ ). Across all participants, the $\mathrm{K}>\mathrm{F}$ contrast was associated with activations in posterior regions. For high-complexity scenes, $\mathrm{K}>\mathrm{F}$ contrast identified activations in the PHG, middle occipital gyrus, and fusiform gyrus. For low-complexity scenes, $\mathrm{K}>\mathrm{F}$ contrast identified activations in the middle temporal gyrus and middle occipital gyrus. Activations in regions responsive to $\mathrm{K}>\mathrm{F}$ did not change with age for either high- or low-complexity scenes.

\section{DISCUSSION}

We found three major influences of scene complexity on human brain function. First, activations in a posterior network including the posterior PHG were greater for high-complexity than lowcomplexity scenes. Second, the difference in activations for highcomplexity versus low-complexity scenes in right posterior PHG grew in magnitude with age. Third, activations in the same right PHG region that were associated with successful memory formation grew with age for high-complexity but not low-complexity scenes. Therefore, functional maturation of right posterior PHG may play a critical role in the development of high-level perception and long-term memory for high-complexity scenes.

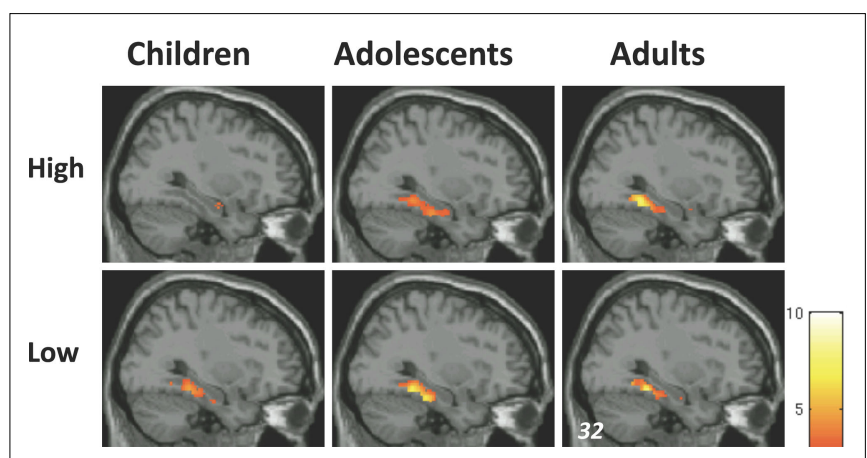

FIGURE 3 | MTL activations associated with successful memory formation ( $R>F$ ) for children, adolescents, and young adults, for highcomplexity scenes (top), and low- complexity scenes (bottom), scenes. Activation maps are overlaid on standard T1 image (for display purposes, $p<0.005$, uncorrected, $T$ value scale presented on the right). Children, $n=16$, 8 male, mean age 10.7, range 8-12 years of age; adolescents, $n=16$, 8 male, mean age 15.9, range 13-17 years of age; adults, $n=14,7$ male, mean age 21.3 , range $19-24$ years of age. This three-group stratification is illustrative, but statistical analyses were conducted with age as a continuum.

\section{IMAGE COMPLEXITY DRIVES POSTERIOR PHG RESPONSE}

The finding that posterior PHG activation was greater for highcomplexity than low-complexity scenes can be related to ideas about how scenes are represented in posterior PHG. In the present study, scenes were categorized by complexity, but there was a strong correlation between the indoor/outdoor and highcomplexity/low-complexity characteristics of the scenes (i.e., if we had analyzed our data with indoor/outdoor factor, we would have found developmental relations between PHG activation and recollection for indoor scenes). Posterior PHG bilaterally is more active when viewing indoor than when viewing outdoor scenes (Henderson et al., 2007), suggesting it may prefer certain types of scene stimuli to others. One explanation for greater posterior PHG activations for indoor scenes is that this region encodes spatial layout information that is primarily defined by the geometric structure in the scene (Epstein and Kanwisher, 1998; Epstein and Ward, 2010). The geometric structure of indoor scenes is more prominent than that of outdoor scene because they include fixed background elements such as walls and windows. However, posterior PHG is also more activated when participants view indoor scenes that contain more rather than fewer objects (Bar et al., 2008), suggesting that the posterior PHG activations observed in our study are not fully explained by the indoor characteristics of the high-complexity scenes. Furthermore, in our and most other studies, the outdoor scenes are nature scenes. If urban or other scenes had been included, then many outdoor scenes would have been high in complexity. Further studies are needed to determine which of these scene dimensions is more relevant for activations observed in posterior PHG.

Since measures of high-level visual properties, such as our measure of scene complexity, tend to correlate with low-level visual properties, such as spatial frequency, we tested if the effects of scene complexity were confounded by low-level visual properties of the scenes, spatial frequency. The activations in PHG remained higher for high-complexity scenes compared to lowcomplexity sense after spatial frequency was statistically controlled, which indicates that the PHG response was driven by a high-level visual property of complexity (number of unique item categories) rather than a low-level visual property (spatial frequency) of the scenes.

\section{FUNCTIONAL MATURATION OF SCENE-COMPLEXITY PERCEPTION}

We found a major developmental influence on the neural correlates of scene-perception that was dependent on scene complexity: scene complexity did not influence the level of activation in posterior PHG in children, but became more pronounced in older participants, suggesting that the specific manner in which posterior PHG supports scene representation undergoes prolonged maturation through young adulthood. There is evidence of maturation in scene-selective regions in the left posterior PHG region (parahippocampal place area, PPA), as evident by larger extent of these regions in adults (Golarai et al., 2007). Scene-selective regions were defined in that study as those that were more active for scenes than for other visual categories (such as objects and faces). In our study, we identified regions in the posterior PHG that were more active for high-complexity scenes than low-complexity scenes. These different approaches of identifying posterior 
PHG activations that relate to scene processing may account for the different lateralization in the developmental effects that are found across the studies.

Scene complexity may be an important dimension in the maturation of scene processing in the brain. High-complexity scenes include more geometric configurations than low-complexity scenes, suggesting that maturational effects may be specific to the processing of geometric configurations. Developmental evidence suggest that although processing of some aspects of geometric information, such as the shape of a room develops early in life (Hermer and Spelke, 1994; Lee and Spelke, 2008), processing of other aspects, such as geometric configuration of objects in space have protracted maturation (Gouteux and Spelke, 2001). It is possible that the processing of these geometric configurations in highcomplexity scenes is limited in children.

An alternative explanation for the maturation of the scene complexity perception is that since high-complexity scenes contained greater numbers of objects, the increased experience with these objects in adults compared to children facilitates the processing of the high-complexity scenes. It is also possible that since highcomplexity scenes contained mostly indoor scenes with man-made objects, whereas low-complexity scenes contained mostly outdoor scenes natural objects, the maturation effect of scene complexity perception may reflect our more innate ability to process natural objects/scenes versus man-made objects/scenes.

\section{DEVELOPMENTAL CHANGES ASSOCIATED WITH FORMING MEMORIES FOR COMPLEX SCENES}

The development of neural correlates of recognition memory for scenes depended on the visual complexity of the scenes. Recollection of high-, but not low-, complexity scenes improved with age. These improvements in the formation of detailed memory for scenes were associated with the functional maturation of right posterior PHG. Activations in right posterior PHG region were greater for subsequently remembered than forgotten scenes across all participants, indicating a role for this region in the formation of memory for scenes. Critically, however, activations in this region increased with age for subsequent memory of high-complexity but not lowcomplexity scenes.

A central question is what aspect of memory encoding changes with age with respect to scene complexity. In the present study, age was specifically associated with the formation of memories that supported subjectively vivid and detailed recollection of more complex visual scenes. Age was not associated with recollection of low-complexity scenes, or familiarity-based recognition of either high- or low-complexity scenes. The specific behavioral relation between age and recollection for high-complexity scenes was paralleled by activations in right posterior PHG that increased with age only for recollection of high-complexity scenes. Furthermore, these age effects in PHG, remained significant after spatial frequency was statistically controlled, which indicates that the age-related changes in MTL activations is driven by a high-level visual property of complexity (number of unique item categories) rather than a low-level visual property (spatial frequency) of the scenes. The convergence of behavioral and imaging findings indicates that the right posterior PHG plays a specific role in the formation of episodic memories that are contextually rich for complex materials.
Interpretation of the selectivity of the developmental influence on MTL-memory relations for high-, but not low-, complexity scenes is constrained by the specific manner in which we defined complexity in our analysis. We defined complexity by counting the number of unique object categories in the scene. The difference between high-and low-complexity scenes could be considered with respect to two perceptual dimensions that may differentially relate to proposed roles of MTL in mnemonic processes. On the one hand, more complex scenes may simply have more object categories and this increase in the sheer number of object categories results in increased demand for detailed representation. Alternatively, it is possible that complex scenes include more spatial relations among the objects and thus may result in increased demand to represent the relations among constituent elements of the scene. Encoding and retrieving detailed contextual information is thought to be supported by the posterior PHG subregions of the MTL, while the representation of relations among the constituent elements of scenes are thought to be specifically subserved by the hippocampus (Cohen et al., 1999; Diana et al., 2007; Eichenbaum et al., 2007). Although these two aspects of mnemonic processes may be complementary, here we show that the age-related effects are unique to the posterior PHG, suggesting that it is the ability to process more contextual details that changes with age. We did not find age-related changes in the hippocampus. The interpretation of the lack of developmental effects in the hippocampus is limited by the nature of the task and the specific stimuli we used. Further studies that manipulate relational memory construction can reveal whether hippocampal activations vary with age under more specific demands for relational memory processes.

The interpretation that immature MTL structure and function limit the episodic specificity of memory formation for complex visual material through development is consistent with several related lines of evidence. First, lesion evidence in the monkey suggests that the posterior PHG play a critical role in memory for relatively demanding associations (Malkova and Mishkin, 2003). In line with its role in supporting the representation of demanding associations, activations in posterior PHG are related to recollection and the encoding and retrieval of contextual information (Diana et al., 2007). Second, maturation in brain regions that support processing of scenes was related to memory performance (Golarai et al., 2007). Here, we were able to document a functional maturation in scene-selective regions and to show that this maturation is directly linked to improvement in recognition memory. This extends the previous findings by Golarai et al. (2007) who correlated measures of the size of scene-selective regions with recognition measures that were taken in an independent session. Third, other evidence for functional changes in MTL contribution have been demonstrated during memory encoding and retrieval. One study of memory encoding showed that connectivity between MTL and PFC in the context of memory encoding changes with age (Menon et al., 2005). Two other studies found developmental changes in MTL activations for recognition memory of verbal material. Activations in anterior left MTL were associated differently with recognition of sentences in older versus younger children (Chiu et al., 2006), and with verbal memory retrieval in adults versus chidren (Paz-Alonso et al., 2008). Our analysis focused on the way in which processing of complex visual material may lead to better recognition. Our results are thus complementary to these previous 
findings and demonstrate that activations in posterior regions in the PHG increase with age when processing complex scenes and that these reduced activations in children may serve as a limiting factor for memory formation in children. Taken together, these findings suggest that protracted maturation in different subregions within the MTL may be evident depending on specific task demands. Future research is needed to determine the developmental trajectory of different subregions within the MTL.

\section{SUMMARY}

Scene complexity modulated activation in a network of regions that are typically involved in scene perception, including a subregion of the MTL, the posterior PHG. Activations in these regions were greater for high- compared to low-complexity scenes. Further, these scene complexity effects increased with age in the right posterior $\mathrm{PHG}$, suggesting that this region undergoes functional maturation with respect to processing of high-complexity scenes. Finally, recollection for high-, but not low-complexity scenes improved with age; recollection for low-complexity scenes and familiarity for high- and low-complexity scenes did not change with age. Activations associated with subsequent memory in the right posterior PHG increased with age for high-, but not low-, complexity scenes. Thus, functional maturation of the PHG appears to be important both for the high-level perception of high-complexity scenes and for the formation of detailed recollection for those scenes. The prolonged functional maturation of this PHG region extends from childhood into young adulthood, and its functional immaturity may limit children and adolescents from forming the detailed and enduring memories for high-complexity scenes that are formed by adults with mature PHG function.

\section{ACKNOWLEDGMENTS}

We thank Aude Oliva for helpful discussions and for providing methods of analyzing image spatial frequency. We thank Antonio Torralba for help with calculating image complexity. We also thank the reviewers for helpful comments. This research was supported by $\mathrm{NIH}$ grant $\mathrm{MH} 08344$.

\section{REFERENCES}

Bar, M., Aminoff, E., and Schacter, D. L. (2008). Scenes unseen: the parahippocampal cortex intrinsically subserves contextual associations, not scenes or places per se. J. Neurosci. 28, 8539-8544.

Billingsley, R. L., Lou Smith, M., and Pat McAndrews, M. (2002). Developmental patterns in priming and familiarity in explicit recollection. J. Exp. Child Psychol. 82, 251-277.

Brewer, J. B., Zhao, Z., Desmond, J. E., Glover, G. H., and Gabrieli, J. D. E. (1998). Making memories: brain activity that predicts how well visual experience will be remembered. Science 281, 1185-1187.

Buckner, R. L., Kelley, W.M., and Petersen, S.E. (1999). Frontal cortex contributes to human memory formation. Nat. Neurosci. 2, 311-314.

Chiu, C.Y. P., Schmithorst, V. J., Brown, R. D., Holland, S. K., and Dunn, S. (2006). Making memories: A cross-sectional investigation of episodic memory encoding in childhood using fMRI. Dev. Neuropsychol. 29, 321-340.

Cohen, N. J., Ryan, J., Hunt, C., Romine, L., Wszalek, T., and Nash, C. (1999). Hippocampal system and declarative (relational) memory: summarizing the data from functional neuroimaging studies. Hippocampus 9, 83-98.

Cycowicz, Y.M., Friedman, D., Snodgrass, J.G., and Duff, M. (2001). Recognition and source memory for pictures in children and adults. Neuropsychologia 39, 255-267.

Davachi, L., Mitchell, J. P., and Wagner, A. D. (2003). Multiple routes to memory: Distinct medial temporal lobe processes build item and source memories. Proc. Natl. Acad. Sci. U.S.A. 100, 2157-2162.

De Valois, R. L., Albrecht, D. G., and Thorell,L.G. (1982).Spatial frequency selectivity of cells in macaque visual cortex. Vision Res. 22, 545-559.

Diamond, R., and Carey, S. (1977). Developmental changes in the representation of faces. J. Exp. Child Psychol. 23, 1-22.

Diana, R. A., Yonelinas, A. P., and Ranganath, C. (2007). Imaging recollection and familiarity in the medial temporal lobe: a three-component model. Trends Cogn. Sci. (Regul. Ed.) $11,379-386$.

Dirks, J., and Neisser, U. (1977). Memory for objects in real scenes: the development of recognition and recall. J. Exp. Child Psychol. 23, 315-328.

Eichenbaum, H., Yonelinas, A. P., and Ranganath, C. (2007). The medial temporal lobe and recognition memory. Annu. Rev. Neurosci. 30, 123-152.

Epstein, R., and Kanwisher, N. (1998). A cortical representation of the local visual environment. Nature 392, 598-601.

Epstein, R.A., and Ward,E.J. (2010). How reliable are visual context effects in the parahippocampal place area? Cereb. Cortex. 20, 294-303.

Feldman, J. (1997). Regularity-based perceptual grouping. Comput. Intell. 13, 582-623.

Foster, K. H., Gaska, J. P., Nagler, M., and Pollen, D. A. (1985). Spatial and temporal frequency selectivity of neurones in visual cortical areas V1 and V2 of the macaque monkey. J. Physiol. 365, 331-363.
Ghetti, S., and Angelini, L. (2008). The development of recollection and familiarity in childhood and adolescence: evidence from the dual-process signal detection model. Child Dev. 79 , 339-358.

Giedd, J.N., Blumenthal, J., Jeffries, N. O., Castellanos, F. X., Liu, H., Zijdenbos, A., Paus, T., Evans, A.C., and Rapoport, J.L. (1999). Brain development during childhood and adolescence: a longitudinal MRI study. Nat. Neurosci. 2, 861-863.

Giedd, J.N., Vaituzis, A. C., Hamburger, S. D., Lange, N., Rajapakse, J. C., Kaysen, D., Vauss, Y. C., and Rapoport, J. L. (1996). Quantitative MRI of the temporal lobe, amygdala, and hippocampus in normal human development: ages 4-18 years. J. Comp. Neurol. 366 , 223-230.

Gogtay, N., Giedd, J.N., Lusk, L., Hayashi, K. M., Greenstein, D., Vaituzis, A. C., Nugent, T. F., Herman, D. H., Clasen, L. S., Toga, A. W., Rapoport, J. L., and Thompson, P. M. (2004). Dynamic mapping of human cortical development during childhood through early adulthood. Proc. Natl. Acad. Sci. U.S.A. 101, 8174-8179.

Gogtay, N., Nugent, T. F. III, Herman, D. H., Ordonez, A., Greenstein, D. Hayashi, K. M., Clasen, L., Toga, A. W., Giedd, J. N., Rapoport, J. L., and Thompson, P. M. (2006). Dynamic mapping of normal human hippocampal development. Hippocampus $16,664-672$.

Golarai, G., Ghahremani, D. G., WhitfieldGabrieli, S., Reiss, A., Eberhardt, J. L., Gabrieli, J.D. E., and Grill-Spector, K. (2007). Differential development of high-level visual cortex correlates with category-specific recognition memory Nat. Neurosci. 10, 512-522.

Gouteux, S., and Spelke, E. S. (2001). Children's use of geometry and landmarks to reorient in an open space. Cognition 81, 119-148.

Henderson, J. M., Larson, C. L., and Zhu, D. C. (2007). Cortical activation to indoor versus outdoor scenes: an fMRI study. Exp. Brain Res. 179, 75-84

Hermer, L., and Spelke, E. S. (1994). A geometric process for spatial reorientation in young children. Nature 370, 57-59.

Huttenlocher, P. R. (1979). Synaptic density in human frontal cortex - developmental changes and effects of aging. Brain Res. 163, 195-205.

Iidaka, T., Yamashita, K., Kashikura, K., and Yonekura, Y. (2004). Spatial frequency of visual image modulates neural responses in the temporooccipital lobe. An investigation with event-related fMRI. Brain Res. Cogn. Brain Res. 18, 196-204.

Jacoby, L. L., Toth, J. P., and Yonelinas, A. P. (1993). Separating conscious and unconscious influences of memory: measuring recollection.J. Exp. Psychol. Gen. 122, 139-154.

Janowsky, J. S., Shimamura, A. P., and Squire, L. R. (1989). Source memory impairment in patients with frontal lobe lesions. Neuropsychologia 27, 1043-1056.

Lee, S. A., and Spelke, E. S. (2008). Children's use of geometry for reorientation. Dev. Sci. 11, 743-749.

Malkova, L., and Mishkin, M. (2003). One-trial memory for object-place associations after separate lesions of hippocampus and posterior parahippocampal region in the monkey. $J$. Neurosci. 23, 1956-1965. 
Mandler, J. M., and Robinson, C. A. (1978). Developmental changes in picture recognition. J. Exp. Child Psychol. 26, 122-136.

Menon, V., Boyett-Anderson, J. M., and Reiss,A.L. (2005). Maturation of medial temporalloberesponse and connectivity during memory encoding. Brain Res. Cogn. Brain Res. 25, 379-385.

Nichols, E. A., Kao, Y.-C., Verfaellie, M., and Gabrieli, J. D. E. (2006). Working memory and long-term memory for faces: evidence from fMRI and global amnesia for involvement of the medial temporal lobes. Hippocampus 16, 604-616.

Ofen, N., Kao, Y.-C., Sokol-Hessner, P., Kim, H., Whitfield-Gabrieli, S., and Gabrieli, J. D. E. (2007). Development of the declarative memory system in the human brain. Nat. Neurosci. 10, 1198-1205.

Oliva, A., Mack, M. L., Shrestha, M., and Peeper, A. (2004). Identifying the perceptual dimensions of visual complexity in scenes. In Proceedings of the 26th Annual Meeting of the Cognitive Science Society., Chicago, August.

Paz-Alonso, P. M., Ghetti, S., Donohue, S. E., Goodman, G. S., and Bunge, S. A. (2008). Neurodevelopmental correlates of true and false recognition. Cereb. Cortex 18, 2208-2216.
Peyrin, C., Baciu, M., Segebarth, C., and Marendaz, C. (2004). Cerebral regions and hemispheric specialization for processing spatial frequencies during natural scene recognition. An event-related fMRI study. Neuroimage 23, 698-707.

Russell, B. C., Torralba, A., Murphy, K. P., and Freeman, W. T. (2008). LabelMe: A database and web-based tool for image annotation. Int. J. Comput. Vis. 77, 157-173.

Schacter, D. L., Harbluk, J. L., and Mclachlan, D. R. (1984). Retrieval without recollection: an experimental analysis of source amnesia. J. Verbal Learn. Verbal Behav. 23, 593-611.

Scoville, W. B., and Milner, B. (1957). Loss of recent memory after bilateral hippocampal lesions. J. Neurol. Neurosurg. Psychiatr. 20, 11-21.

Sowell, E. R., and Jernigan, T. L. (1998). Further MRI evidence of late brain maturation: limbic volume increases and changing asymmetries during childhood and adolescence. Dev. Neuropsychol. 14, 599-617.

Sowell, E. R., Thompson, P. M., Leonard, C. M., Welcome, S. E., Kan, E., and Toga, A. W. (2004). Longitudinal mapping of cortical thickness and brain growth in normal children. $J$. Neurosci. 24, 8223-8231.
Sowell,E. R., Trauner, D. A., Gamst, A., and Jernigan, T. L. (2002). Development of cortical and subcortical brain structures in childhood and adolescence: a structural MRI study. Dev. Med. Child Neurol. 44, 4-16.

Squire, L. R. (1992). Memory and the hippocampuss: a synthesis from findings with rats, monkeys, and humans. Psychol. Rev. 99, 195-231.

Torralba, A., and Oliva, A. (2003). Statistics of natural image categories. Network: Comput. Neural Syst. 14, 391-412.

Vuilleumier, P., Armony, J. L., Driver, J. and Dolan, R. J. (2003). Distinct spatial frequency sensitivities for processing faces and emotional expressions. Nat. Neurosci. 6, 624-631.

Wagner, A. D., Schacter, D. L., Rotte, M., Koutstaal, W., Maril, A., Dale, A. M., Rosen, B. R., and Buckner, R. L. (1998). Building memories: remembering and forgetting of verbal experiences as predicted by brain activity. Science 281 , 1188-1191.

Yonelinas, A. P. (1999). The contribution of recollection and familiarity to recognition and source-memory judgments: a formal dual-process model and an analysis of receiver operating characteristics. J. Exp. Psychol. Learn. Mem. Cogn. 25, 1415-1434.
Zola-Morgan, S., Squire, L. R., and Amaral, D. G. (1986). Human amnesia and the medial temporal region: enduring memory impairment following a bilateral lesion limited to field CA1 of the hippocampus. J. Neurosci. 6, 2950-2967.

Conflict of Interest Statement: The authors declare that the research was conducted in the absence of any commercial or financial relationships that could be construed as a potential conflict of interest.

Received: 01 September 2009; paper pending published: 10 October 2009; accepted: 16 February 2010; published online: 05 March 2010

Citation: Chai XJ, Ofen N, Jacobs LF and Gabrieli JDE (2010) Scene complexity: Influence on perception, memory, and development in the medial temporal lobe. Front. Hum. Neurosci. 4:21. doi: 10.3389/fnhum.2010.00021

Copyright (C) 2010 Chai, Ofen, Jacobs and Gabrieli. This is an open-access article subject to an exclusive license agreement between the authors and the Frontiers Research Foundation, which permits unrestricted use, distribution, and reproduction in any medium, provided the original authors and source are credited. 